\title{
TRATAMENTO DE SEMENTES DE MILHO PARA CONTROLAR Dichelops melacanthus EM DIFERENTES TIPOS DE SOLO
}

\author{
CARLOS BRUSTOLIN ${ }^{1}$, PEDRO MANUEL OLIVEIRA JANEIRO NEVES ${ }^{2}$, \\ RODOLFO BIANCO ${ }^{3}$ e ORCIAL CEOLIN BORTOLOTTO ${ }^{2}$
}

\begin{abstract}
${ }^{1}$ Msc. Eng. Agrônomo, Sumitomo Chemical Latin America/SCLA, Londrina, Paraná, Brasil, cbrustolin@sumitomo-chem.com.br; ${ }^{2}$ Universidade Estadual de Londrina, Avenida Celso Garcia Cid, Rodovia Celso Garcia Cid - Pr $445 \mathrm{Km} 380, \mathrm{~s} / \mathrm{n}$, Campus Universitário,CEP: 86057-970,Londrina,Paraná,Brasil,pedroneves@uel.br, bortolotto.orcial@gmail.com; ${ }^{3}$ Instituto Agronômico do Paraná, Rodovia Celso Garcia Cid, km 375, CEP: 86047-902, Londrina, Paraná, Brasil, rbianco@iapar.br
\end{abstract}

Revista Brasileira de Milho e Sorgo, v.16, n.1, p. 13-21, 2017

\begin{abstract}
RESUMO - O tratamento de sementes (TS) é a principal técnica empregada para o controle de Dichelops melacanthus (Hemiptera: Pentatomidae) em milho. Entretanto, acredita-se que a eficiência desta estratégia pode ser influenciada pelo tipo de solo no qual a lavoura é cultivada. Desse modo, este trabalho comparou a eficiência do TS dos inseticidas [tiametoxam e imidacloprido + tiodicarbe] em relação a duas testemunhas (com infestação e sem infestação) em quatro tipos de solo: Latossolo Vermelho distroférrico (LVdf); Latossolo Vermelho Amarelo arenoso (LVA arenoso); Latossolo Vermelho Amarelo argiloso (LVA argiloso) e Latossolo Vermelho distrófico (LVd). De forma geral, ambos os TS reduziram os danos da praga em todos os tipos de solo, quando comparados às plantas infestadas com $D$. melacanthus e sem TS. Por outro lado, o pior desempenho de ambos os TS foi observado no LVA arenoso, em que as plantas apresentaram menor comprimento de espiga e menor produtividade. Este estudo demonstrou que a eficiência do TS com tiametoxam, assim como imidacloprido + tiodicarbe, é afetada pelo tipo de solo. Desse modo, maiores cuidados devem ser tomados no cultivo de lavouras de milho em regiões com solos arenosos, e a adoção de estratégia complementar pode ser necessária.
\end{abstract}

Palavras-chave: percevejo-barriga-verde, pragas do milho, pragas iniciais, neonicotinoides, adsorção de inseticidas.

\section{SEED CORN TREATMENT FOR Dichelops melacanthus CONTROL IN DIFFERENT SOIL TYPES}

\begin{abstract}
The seed treatment (ST) is the main tool used to control Dichelops melacanthus (Hemiptera: Pentatomidae) in maize fields. However, the soil type in which the plants are developed probably influences the efficiency of this strategy. In this sense, this study aimed to evaluate the performance of two insecticides (ST) [thiamethoxam (52.5 g.i.a); imidacloprid + thiodicarb $(45+135)$ g.i.a] in relation to control (infested and non-infested plants) for D. melacanthus control in four types of soil: Dystroferric Red Latosol (DRL), sandy Red-Yellow Latosol (sandy RYL); clay Red-Yellow Latosol (clay RYL); and dystrophic Red Latosol (dystrophic RL). In general, ST reduced the pest damage in all soil types. In the other hand, the development plants in sandy RYL were hampered, showing a shorter length of ear and lower yield in both ST. This study showed that the ST performance with thiamethoxam and imidacloprid + thiodicarb is affected by the soil type. In this sense, maize fields cultivated in sand soils requires a careful management, and complementary strategies can be necessary.
\end{abstract}

Keywords: green-belly stinkbug, maize pests, initial pests, neonicotinoides, insecticide adorsoption. 
O percevejo-barriga-verde Dichelops melacanthus (Hemiptera: Pentatomidae) tem ocasionado muitas preocupações aos produtores de milho, em razão da sua capacidade de dano. No Brasil, essa espécie ganhou maior importância após a consolidação do sistema plantio direto, que propicia condições favoráveis para o desenvolvimento da praga (Chocorosqui \& Panizzi, 2004). Atualmente, a distribuição dessa espécie no país ocorre principalmente em lavouras cultivadas entre o norte do Paraná e o Centro-Oeste. O dano em milho é ocasionado pela fase adulta de D. melacanthus (Chocorosqui \& Panizzi, 2008), prejudicando o desenvolvimento normal das plântulas de milho (Roza-Gómes et al., 2011), e a produtividade pode ser comprometida caso nenhuma estratégia de controle seja adotada (Brustolin et al., 2011). Desse modo, em áreas com histórico de ocorrência da praga, recomenda-se a adoção do tratamento de sementes (TS), que tem sido uma tática de controle eficiente na redução dos danos da praga (Brustolin et al., 2011).

Recentemente, alguns trabalhos têm demonstrado que a adsorção de inseticidas é influenciada por fatores químicos e físicos (textura) do solo com o qual esse agrotóxico está em contato (Kodešová et al., 2011; Tabassum et al., 2014). Porém, esses estudos têm sido desenvolvidos para avaliar o potencial de impacto ambiental, e pouco se conhece a respeito da eficiência de diferentes i.a. para o controle de pragas nessas diferentes condições (tipos de solo). Nesse sentido, acredita-se que a eficiência dos inseticidas utilizados no TS para o controle de pragas iniciais pode ser influenciada pela textura do tipo de solo onde a lavoura será cultivada. Assim, a obtenção desta informação é importante para melhor compreender a relação solo, planta e inseto, além de possibilitar recomendações mais adequadas para os agricultores, permitindo um maior sucesso no combate da praga -alvo. Em razão da escassez de informações sobre esta relação, este estudo objetivou avaliar a influência da textura de diferentes tipos de solo sobre o desempenho do TS em milho para o controle de D. melacanthus.

\section{Material e Métodos}

\section{Local do estudo}

O estudo foi realizado na área experimental do Instituto Agronômico do Paraná (IAPAR), em Londrina, Paraná $\left(23^{\circ} 19^{\prime} 44^{\prime \prime}\right.$ S; 51 $\left.1^{\circ} 12^{\prime} 10,23^{\prime \prime} \mathrm{O}\right)$, no período entre abril e setembro de 2011 (segunda safra de milho).

\section{Tipos de solo avaliados}

Para o estudo, foram avaliados quatro tipos de solo (Tabela 1), segundo a classificação da Embrapa (Santos et al., 2006). O solo foi coletado no ano 2004 e foi depositado em um canteiro de alvenaria ( $10 \mathrm{x} 1 \mathrm{x}$ 0,7 metros, de comprimento, largura e profundidade, respectivamente) na área experimental do IAPAR.

\section{Procedência dos adultos de $D$. melacanthus utilizados no estudo}

As posturas de D. melacanthus para a realização do experimento foram obtidas no laboratório de criação de insetos do IAPAR - Londrina, PR. A criação dos insetos ocorreu com a oferta de dieta natural composta por grãos de soja, amendoim, girassol e vagens de feijão. Esses indivíduos desenvolveramse em condições controladas de temperatura $\left(25 \pm 1^{\circ} \mathrm{C}\right)$, umidade relativa $(60 \pm 10 \%)$ e fotofase (14 horas). 
Tabela 1. Características físico-químicas dos quatro tipos de solo utilizados no estudo. IAPAR, Londrina, Paraná, 2011.

\begin{tabular}{ccccc}
\hline $\begin{array}{c}\text { Análise química } \\
\text { do solo }\end{array}$ & $\begin{array}{c}\text { Latossolo Vermelho } \\
\text { distroférrico }\end{array}$ & $\begin{array}{c}\text { Latossolo Vermelho } \\
\text { Amarelo arenoso }\end{array}$ & $\begin{array}{c}\text { Latossolo Vermelho } \\
\text { Amarelo argiloso }\end{array}$ & $\begin{array}{c}\text { Latossolo } \\
\text { Vermelho } \\
\text { distrófico }\end{array}$ \\
\hline \% M.O. & 1,95 & 1,21 & 2,05 & 2,18 \\
$\mathrm{pH}$ & 5,43 & 5,34 & 5,58 & 5,56 \\
CTC $\left(\mathrm{cMol} / \mathrm{dm}^{3}\right)$ & 11,72 & 6,16 & 10,07 & 9,34 \\
\hline \multirow{2}{*}{ Análise física } & Latossolo Vermelho & Latossolo Vermelho & Latossolo Vermelho & Latossolo \\
do solo & distroférrico & Amarelo arenoso & Amarelo argiloso & Vermelho \\
& \multicolumn{2}{c}{ distrófico } \\
\hline Argila & 78 & 17 & 66 & 40 \\
\% Areia & 10 & 80 & 25 & 54 \\
\hline
\end{tabular}

\section{Semeadura, manejo nutricional e irrigação das plantas de milho}

Para o estudo, foi utilizado o híbrido de milho P30F80 Hx, que foi semeado manualmente na profundidade média de $4 \mathrm{~cm}$, com densidade de seis sementes/metro linear. A adubação química foi realizada em duas etapas. A adubação de base ocorreu com o emprego de $400 \mathrm{Kg} / \mathrm{ha}$ de NPK (08-28-16), seguida de uma adubação de cobertura aos 20 dias após a emergência (DAE), aplicando-se $300 \mathrm{Kg} / \mathrm{ha}$ de $\mathrm{Ca}\left(\mathrm{NO}_{3}\right)^{2}$. Por não ter ocorrido precipitação nos primeiros $30 \mathrm{DAE}$, durante este período foi realizado a irrigação manual.

\section{Delineamento experimental}

\section{e inseticidas utilizados no TS de milho}

A unidade experimental (repetição) foi composta por uma caixa plástica (sem o fundo) com três plantas de milho. Sobre a caixa foi colocada uma gaiola "telada" (57 x 25 x $80 \mathrm{~cm}$ de comprimento, largura e altura, respectivamente) com as extremidades laterais afixadas ao solo. A infestação com percevejos foi realizada colocando-se cinco adultos de D. melacanthus em cada gaiola, em razão de ser uma densidade suficiente para ocasionar danos na cultura (Rodrigues, 2011). O TS foi realizado com dois inseticidas: tiametoxam 350 FS (52,5 g. i.a./60.000 sementes) e imidacloprido + tiodicarbe $600(150+450) \mathrm{SC}(45$ + 135 g. i.a./60.000 sementes). Complementarmente foram utilizadas duas testemunhas (sementes não tratadas), sendo uma realizada com infestação (C/I) e outra sem infestação (S/I), para cada tipo de solo. $\mathrm{O}$ estudo foi realizado em delineamento experimental de blocos ao acaso, em esquema fatorial 2 (i.a. de inseticidas) x 4 (tipos de solo) com quatro repetições.

\section{Infestações das gaiolas com adultos} de D. melacanthus

Para o estudo foram realizadas duas infestações, permitindo-se a alimentação por sete dias em cada infestação (totalizando 14 dias). A primeira infestação foi realizada aos 2 DAE do milho (estádio fenológico $\mathrm{V}_{\mathrm{E}}$ ) (adaptado de Ritchie \& Hanway, 1989), possibilitando avaliar o impacto inicial do TS sobre os adultos de D. melacanthus. As mesmas plan- 
tas foram novamente infestadas aos 9 DAE do milho (estádio $\mathrm{V}_{2}$ ) (adaptado de Ritchie \& Hanway, 1989), para avaliar o efeito residual de ambos os TS.

\section{Eficiência do TS e intensidade de dano nas plantas de milho}

A mortalidade dos insetos foi avaliada aos 3 e 7 dias após a infestação (DAI). Durante as avaliações, os insetos mortos foram quantificados e retirados da unidade experimental. $\mathrm{Na}$ testemunha $\mathrm{C} / \mathrm{I}$ foi mantida sempre a quantia de cinco insetos/parcela, e não ocorreu mortalidade de percevejos neste tratamento.

A injúria ocasionada por D. melacanthus em plantas de milho foi quantificada utilizando-se uma escala de notas, onde: $0=$ planta sem sintoma de ataque; 1 = planta com pequenas pontuações amareladas nas folhas, sem redução de porte; 2 = planta com pontuações amareladas pronunciadas nas folhas do cartucho e pequena redução de porte; nota 3 = planta com cartucho "encharutado" ou planta perfilhada; 4 = planta com cartucho fortemente destruído e/ou com forte redução de porte (adaptado de Chocorosqui, 2001).

Essas notas foram multiplicadas pelos respectivos pesos: nota $0=$ peso 0 ; nota $1=$ peso 0,5 ; nota $2=$ peso 1 ; nota $3=$ peso 2 e nota $4=$ peso 3 , permitindo assim o cálculo da intensidade de dano na planta (ID= nota $\mathrm{x}$ peso). Em razão de cada parcela ser constituída por três plantas, foi calculada a ID média por parcela, seguindo a fórmula: $\sum$ [ID na planta $1+$ ID planta $2+$ ID planta 3] / 3.

\section{Avaliação do desenvolvimento vegetativo e dos componentes de produção das plantas de milho}

Durante o estudo também foi realizada a medição da espessura $(\mathrm{cm})$ do colmo das plantas, aos 119
DAE. Para isso, foi utilizado um paquímetro que foi afixado ligeiramente acima do segundo nó da planta. Com o uso de uma trena, foi avaliada a altura de plantas, considerando-se o comprimento entre o nível do solo e a última folha curvada da planta, aos 151 DAE.

A colheita do milho foi realizada aos 160 DAE. Nessa etapa foram avaliados os seguintes parâmetros: comprimento de espiga (distância da base da espiga até o último grão da ponta); número de fileiras de grãos na espiga; número de grãos por espiga e produtividade (g/parcela). Para o cálculo da produtividade foi realizada a correção da umidade de grãos de milho para $14,5 \%$.

\section{Análises estatísticas}

Os dados foram submetidos a análises exploratórias para avaliar as pressuposições de normalidade e independência dos resíduos, a homogeneidade de variância dos tratamentos e a aditividade do modelo para permitir a aplicação da análise de variância (ANOVA). As comparações múltiplas de médias foram realizadas pelo teste de Tukey $(p \leq 0,05)$, em análise conjunta dos dados quando houve interação. Quando não houve interação TS x tipo de solo, comparou-se apenas a eficiência do TS para cada tipo de solo em particular. Os dados de porcentagem foram transformados utilizando-se a fórmula arcsen $\left[(\mathrm{x} / 100)^{1 / 2}\right]$.

\section{Resultados}

De forma geral, ambos os i.a., tiametoxam e imidacloprido + tiodicarbe, foram eficientes na proteção das plantas de milho infestadas aos 2 DAE, com mortalidade similar de D. melacanthus em ambos os tratamentos de sementes. Na infestação realizada aos 
9 DAE, os resultados também foram semelhantes, exceto no Latossolo Vermelho distroférrico, em que foi observada maior mortalidade para tiametoxam (Tabela 2). As sementes tratadas refletiram também em menor intensidade de dano em todos os tipos de solo, sendo que para o Latossolo Vermelho distroférrico e o Latossolo Vermelho Amarelo arenoso o TS com tiametoxam apresentou os menores índices de danos (Tabela 2).

Os maiores danos ocasionados por D. melacanthus ocorreram nas plantas desenvolvidas no Latossolo Vermelho Amarelo arenoso (Tabelas 2 e 3). Nesse solo, embora o TS tenha reduzido os danos da praga, observou-se menor comprimento de espiga, e também menor produtividade do que as plantas desenvolvidas nos outros tipos de solo (Tabela 3). O maior impacto de $D$. melacanthus nas plantas de milho desenvolvidas nesse solo foi ainda mais evidente na testemunha (com infestação), com elevada intensidade de dano, e também menor diâmetro de colmo, altura de planta, comprimento de espiga, número de sementes por espiga e produtividade (Tabelas 2 e 3 ).

\section{Discussão}

Os neonicotinoides utilizados no presente estudo, tiametoxam e imidacloprido, são reconhecidamente inseticidas altamente solúveis em condições de solo úmido (California Department of Pesticide Regulation, 2006; University of Hertfordshire, 2013). Assim, essa condição permite que as plantas absorvam rapidamente os i.a., mantendo-se protegidas do ataque de pragas, conforme demonstrado no presente estudo (Tabela 2). Entretanto, nosso trabalho demonstrou que o tipo de solo no qual a planta se desenvolve poderá comprometer o desempenho de ambos os TS. Isso foi comprovado pelo contraste dos resultados, em que o TS nas plantas desenvolvidas em Latossolo Vermelho distroférrico, Latossolo Vermelho Amarelo argiloso e Latossolo Vermelho distrófico refletiu em melhor desempenho e produtividade das plantas comparativamente ao Latossolo Vermelho Amarelo arenoso.

A intensidade de dano para as plantas com TS foi considerada baixa em todos os tipos de solo, uma vez que as notas indicaram apenas pontuações na área foliar do milho, as quais são classificadas como danos leves (Chocorosqui, 2001). Apesar disso, a intensidade de dano foi significativamente menor para o tiametoxam nas plantas desenvolvidas no Latossolo Vermelho distroférrico e Latossolo Vermelho Amarelo arenoso. Essa diferença pode ter sido em razão da menor adsorção do tiametoxam às partículas de solo, conforme já demonstrado por Campbell et al. (2005). Esse fato ocorre provavelmente por causa da fraca ligação do tiametoxam ao carbono orgânico do solo (Carbo et al., 2007). Entretanto, essa sutil diferença não se refletiu nos parâmetros produtivos, e ambos os TS apresentaram desempenho similar na proteção das plantas de milho.

De forma geral, embora a eficiência do TS e o índice de dano tenham sido similares entre os diferentes tipos de solo, o TS no Latossolo Vermelho Amarelo arenoso apresentou a menor produtividade entre os quatro tipos de solo. Desse modo, acreditase que a menor fertilidade deste solo, associada ao elevado teor de areia (80\%), possa ter comprometido a recuperação das plantas após a infestação da praga. Essa observação foi ainda mais evidente na testemunha (com infestação), que de forma geral apresentou os piores parâmetros de desenvolvimento das plantas, indicando uma menor capacidade de recuperação nessa condição. De acordo com Roza-Gómes et al. (2011), a infestação de um adulto de D. melacanthus 
Tabela 2. Impacto do tratamento de sementes de milho (P30F80 Hx) sobre D. melacanthus e desenvolvimento das plantas de milho em diferentes tipos de solo. IAPAR, Londrina, Paraná, 2011.

\begin{tabular}{|c|c|c|c|c|c|c|c|c|}
\hline \multicolumn{9}{|c|}{ Parâmetros avaliados em cada tipo de solo } \\
\hline \multirow{5}{*}{ Tratamento } & \multicolumn{2}{|c|}{ Latossolo } & \multicolumn{2}{|c|}{ Latossolo } & \multicolumn{2}{|c|}{ Latossolo } & \multicolumn{2}{|c|}{ Latossolo } \\
\hline & \multicolumn{2}{|c|}{ Vermelho } & \multicolumn{2}{|c|}{ Vermelho } & \multicolumn{2}{|c|}{ Vermelho } & \multicolumn{2}{|c|}{ Vermelho } \\
\hline & \multicolumn{2}{|c|}{ distroférrico } & \multicolumn{2}{|c|}{ Amarelo arenoso } & \multicolumn{2}{|c|}{ Amarelo argiloso } & \multicolumn{2}{|c|}{ distrófico } \\
\hline & \multicolumn{8}{|c|}{ Mortalidade de D. melacanthus ${ }^{1,3}$} \\
\hline & $2 \mathrm{DAE}$ & $9 \mathrm{DAE}$ & $2 \mathrm{DAE}$ & $9 \mathrm{DAE}$ & $2 \mathrm{DAE}$ & $9 \mathrm{DAE}$ & $2 \mathrm{DAE}$ & $9 \mathrm{DAE}$ \\
\hline Tiametoxam & $90 \pm 4,1 \mathrm{a}$ & $65 \pm 9,3 \mathrm{a}$ & $100 \pm 0 \mathrm{a}$ & $50 \pm 7,9 \mathrm{a}$ & $85 \pm 9,7 \mathrm{a}$ & $50 \pm 2,1 \mathrm{a}$ & $85 \pm 10,2 \mathrm{a}$ & $40 \pm 9,1 \mathrm{a}$ \\
\hline $\begin{array}{c}\text { Imidacloprido + } \\
\text { Tiodicarbe }\end{array}$ & $85 \pm 8,3$ a & $30 \pm 5,4 \mathrm{~b}$ & $95 \pm 7,1 \mathrm{a}$ & $25 \pm 5,2 \mathrm{a}$ & $75 \pm 8,8$ a & $30 \pm 2,1 \mathrm{a}$ & $85 \pm 11,1 \mathrm{a}$ & $40 \pm 5,1 \mathrm{a}$ \\
\hline C.V. $\%$ & 12,8 & 31,6 & 8,9 & 55,0 & 31,2 & 86,6 & 30,6 & 86,6 \\
\hline Tratamento & \multicolumn{8}{|c|}{ Intensidade de dano (ID) ${ }^{2}$ (22 DAE) } \\
\hline Tiametoxam & \multicolumn{2}{|c|}{$0,12 \pm 0,1$ a $\mathrm{A}$} & \multicolumn{2}{|c|}{$0,29 \pm 0,0$ a $\mathrm{A}$} & \multicolumn{2}{|c|}{$0,71 \pm 0,1$ a $\mathrm{A}$} & \multicolumn{2}{|c|}{$0,25 \pm 0,1$ a $\mathrm{A}$} \\
\hline $\begin{array}{c}\text { Imidacloprido }+ \\
\text { Tiodicarbe }\end{array}$ & \multicolumn{2}{|c|}{$1,08 \pm 0,3 \mathrm{~b} \mathrm{~A}$} & \multicolumn{2}{|c|}{$1,95 \pm 0,3 \mathrm{~b} A$} & \multicolumn{2}{|c|}{$1,2 \pm 0,5$ a $\mathrm{A}$} & \multicolumn{2}{|c|}{$0,83 \pm 0,4$ a $\mathrm{A}$} \\
\hline Testemunha $\mathrm{C} / \mathrm{I}$ & \multicolumn{2}{|c|}{$7,66 \pm 1,4$ c A } & \multicolumn{2}{|c|}{$10,16 \pm 2,5$ c B } & \multicolumn{2}{|c|}{$9,02 \pm 1,7 \mathrm{~b} \mathrm{AB}$} & \multicolumn{2}{|c|}{$7,67 \pm 2,1 \mathrm{~b} \mathrm{~A}$} \\
\hline C.V. $\%$ & \multicolumn{8}{|c|}{14,9} \\
\hline Tratamento & \multicolumn{8}{|c|}{ Altura de plantas $(\mathrm{cm})(119 \mathrm{DAE})^{2}$} \\
\hline Tiametoxam & \multicolumn{2}{|c|}{$163,75 \pm 10,1$ a $\mathrm{A}$} & \multicolumn{2}{|c|}{$160,00 \pm 20,8$ a A } & \multicolumn{2}{|c|}{$135,50 \pm 12,4$ a A } & \multicolumn{2}{|c|}{$163,75 \pm 12,8$ a $\mathrm{A}$} \\
\hline $\begin{array}{c}\text { Imidacloprido }+ \\
\text { Tiodicarbe }\end{array}$ & \multicolumn{2}{|c|}{$157,00 \pm 20,3$ a $\mathrm{A}$} & $153,25 \pm$ & 4 a $\mathrm{AB}$ & $91,7 \pm$ & a B & 152,7 & 3,3 a $\mathrm{AB}$ \\
\hline Testemunha $\mathrm{C} / \mathrm{I}$ & 117,25 &, $1 \mathrm{~b} \mathrm{~A}$ & $59,75 \pm$ & $4 \mathrm{~b} \mathrm{~B}$ & $83,75 \pm 1$ & $\mathrm{~b} \mathrm{AB}$ & 98,0 & $1,5 \mathrm{~b} \mathrm{~A}$ \\
\hline Testemunha S/I & 163,50 &, 4 a $\mathrm{A}$ & $153,00=$ &, 3 a $\mathrm{A}$ & $132,25 \pm$ & ,4 a A & 153,2 & 4,3 a $\mathrm{A}$ \\
\hline C.V. $\%$ & & & & & & & & \\
\hline
\end{tabular}

\begin{tabular}{|c|c|c|c|c|}
\hline \multirow{2}{*}{$\begin{array}{c}\text { Tratamento } \\
\text { Tiametoxam }\end{array}$} & \multicolumn{4}{|c|}{ Diâmetro do colmo $(\mathrm{cm})(151 \mathrm{DAE})^{2}$} \\
\hline & $2,04 \pm 0,5$ a $\mathrm{A}$ & $2,35 \pm 0,8$ a $A$ & $1,91 \pm 0,8$ a $\mathrm{A}$ & $2,27 \pm 0,7$ a $A$ \\
\hline $\begin{array}{l}\text { Imidacloprido }+ \\
\text { Tiodicarbe }\end{array}$ & $1,93 \pm 0,1$ a $\mathrm{A}$ & $2,04 \pm 0,3$ a $A$ & $1,67 \pm 0,8$ a $A$ & $1,96 \pm 0,4$ a $A$ \\
\hline Testemunha C/I & $1,31 \pm 0,4 \mathrm{~b} \mathrm{~A}$ & $0,88 \pm 0,1 \mathrm{~b} \mathrm{~B}$ & $1,07 \pm 0,4 \mathrm{~b} \mathrm{AB}$ & $1,24 \pm 0,3 \mathrm{~b} \mathrm{AB}$ \\
\hline Testemunha S/I & $2,21 \pm 0,7$ a $A$ & $2,07 \pm 0,1$ a $A$ & $1,86 \pm 0,5$ a $A$ & $2,05 \pm 0,6$ a A \\
\hline
\end{tabular}

$$
\text { C.V. \% }
$$

11,2

${ }^{1}$ Médias seguidas de mesma letra, minúscula nas colunas e maiúsculas nas linhas, não diferem entre si pelo teste Tukey a $5 \%$ de probabilidade com análise estatística conjunta dos dados. ${ }^{2}$ Médias seguidas de mesma letra, minúscula nas colunas e maiúsculas nas linhas, não diferem entre si pelo teste Tukey a $5 \%$ de probabilidade. ${ }^{3}$ Dados transformados em raiz de $\mathrm{X}+0,5$. Legenda: $\mathrm{C} / \mathrm{I}=$ com infestação; S/I = sem infestação. 
Tabela 3. Componentes de produção de milho (P30F80 Hx) com tratamento de sementes em diferentes tipos de solo. IAPAR, Londrina, Paraná, 2011.

Parâmetros avaliados em cada tipo de solo

Tratamento

Latossolo Vermelho Latossolo Vermelho

Latossolo Vermelho

Latossolo Vermelho distroférrico

Amarelo arenoso

Amarelo argiloso distrófico

Comprimento de espiga $(\mathrm{cm})^{2}$

\begin{tabular}{|c|c|c|c|c|}
\hline Tiametoxam & $11,83 \pm 3,4$ a $\mathrm{AB}$ & $9,67 \pm 2,1$ a B & $13,58 \pm 2,5$ a $A$ & $12,38 \pm 2,8$ a $\mathrm{AB}$ \\
\hline $\begin{array}{c}\text { Imidacloprido + } \\
\text { Tiodicarbe }\end{array}$ & $10,70 \pm 2,8 \mathrm{a} \mathrm{AB}$ & $8,17 \pm 1,8$ a B & $11,71 \pm 1,9$ a $\mathrm{A}$ & $10,79 \pm 1,7$ a $\mathrm{AB}$ \\
\hline Testemunha $\mathrm{C} / \mathrm{I}$ & $5,29 \pm 2,1$ b A & $2,42 \pm 2,3 \mathrm{~b} \mathrm{~A}$ & $2,83 \pm 1,1 \mathrm{~b} \mathrm{~A}$ & $4,42 \pm 2,1 \mathrm{~b} \mathrm{~A}$ \\
\hline Testemunha S/I & $12,75 \pm 3,2$ a $A$ & $10,33 \pm 1,9$ a $\mathrm{A}$ & $11,79 \pm 2,1$ a $\mathrm{A}$ & $11,58 \pm 1,2$ a $A$ \\
\hline
\end{tabular}

\begin{tabular}{|c|c|c|c|c|}
\hline \multirow{2}{*}{$\begin{array}{c}\text { Tratamento } \\
\text { Tiametoxam }\end{array}$} & \multicolumn{4}{|c|}{ Número de fileiras de grãos/espiga ${ }^{1}$} \\
\hline & $13,16 \pm 2,1 \mathrm{a}$ & $13,16 \pm 1,1 \mathrm{a}$ & $13,83 \pm 1,2 \mathrm{a}$ & $13,66 \pm 1,6 \mathrm{a}$ \\
\hline $\begin{array}{c}\text { Imidacloprido + } \\
\text { Tiodicarbe }\end{array}$ & $13,67 \pm 2,8$ a & $13,00 \pm 1,9 \mathrm{a}$ & $14,50 \pm 3,1 \mathrm{a}$ & $14,33 \pm 2,2 \mathrm{a}$ \\
\hline Testemunha C/I & $8,33 \pm 2,2 \mathrm{~b}$ & $3,67 \pm 1,8 b$ & $6,08 \pm 1,1 \mathrm{~b}$ & $7,25 \pm 1,1 \mathrm{~b}$ \\
\hline Testemunha S/I & $13,33 \pm 2,3 \mathrm{a}$ & $14,00 \pm 1,3 \mathrm{a}$ & $13,67 \pm 2,1 \mathrm{a}$ & $13,50 \pm 2,2 \mathrm{a}$ \\
\hline C.V. \% & 3,0 & 10,0 & 17,9 & 13,3 \\
\hline Tratamento & \multicolumn{4}{|c|}{ Número de sementes/espiga $^{2}$} \\
\hline Tiametoxam & $335,33 \pm 22,8$ a A & $273,92 \pm 23,1$ a A & $412,50 \pm 81,2$ a A & $371,42 \pm 45,2$ a A \\
\hline $\begin{array}{c}\text { Imidacloprido }+ \\
\text { Tiodicarbe }\end{array}$ & $339,17 \pm 31,4 \mathrm{a} \mathrm{AB}$ & $189,42 \pm 32,8$ a B & $392,58 \pm 45,7$ a A & $334,25 \pm 39,8$ a $\mathrm{AB}$ \\
\hline Testemunha C/I & $113,75 \pm 19,9$ b A & $36,00 \pm 12,2 \mathrm{~b} \mathrm{~B}$ & $45,84 \pm 13,1 \mathrm{~b} \mathrm{~B}$ & $90,50 \pm 23,1 \mathrm{~b} \mathrm{AB}$ \\
\hline Testemunha S/I & $375,67 \pm 42,3$ a A & $285,50 \pm 29,1$ a A & $360,83 \pm 29,5$ a A & $356,42 \pm 38,6$ a A \\
\hline C.V. $\%$ & \multicolumn{4}{|c|}{17,9} \\
\hline Tratamento & \multicolumn{4}{|c|}{ Produtividade [g/parcela (três plantas) $]^{2}$} \\
\hline Tiametoxam & $324,44 \pm 43,1 \mathrm{a} A \mathrm{~B}$ & $230,10 \pm 43,1$ a B & $390,77 \pm 54,2$ a A & $332,17 \pm 42,8$ a $\mathrm{AB}$ \\
\hline $\begin{array}{c}\text { Imidacloprido }+ \\
\text { Tiodicarbe }\end{array}$ & $287,95 \pm 24,5$ a A & $148,05 \pm 23,4$ a B & $354,32 \pm 42,3$ a A & $326,52 \pm 25,6$ a A \\
\hline Testemunha C/I & $88,52 \mathrm{~b} \pm 22,8 \mathrm{~b} \mathrm{~A}$ & $17,18 \pm 11,6$ b B & $49,52 \pm 21,1 \mathrm{~b} \mathrm{AB}$ & $73,50 \mathrm{~b} \pm 23,1 \mathrm{AB}$ \\
\hline Testemunha S/I & $343,08 \pm 52,1$ a A & $217,16 \pm 36,1$ a $\mathrm{A}$ & $337,29 \pm 34,2$ a A & $317,03 \pm 36,3$ a A \\
\hline C.V. $\%$ & \multicolumn{4}{|c|}{16,9} \\
\hline
\end{tabular}

${ }^{1}$ Médias seguidas de mesma letra, minúscula nas colunas e maiúsculas nas linhas, não diferem entre si pelo teste Tukey a 5\% de probabilidade com análise estatística conjunta dos dados. ${ }^{2}$ Médias seguidas de mesma letra, minúscula nas colunas e maiúsculas nas linhas, não diferem entre si pelo teste Tukey a 5\% de probabilidade. Legenda: C/I = com infestação; S/I = sem infestação. 
por planta de milho (entre o estádio V1 até o V3) reduz a massa seca das raízes em 27,3\%. Embora o presente estudo não tenha avaliado este parâmetro, pode-se sugerir que a infestação utilizada (equivalente a 1,66 adultos/planta) tenha reduzido significativamente o sistema radicular das plantas, e o fato de o Latossolo Vermelho Amarelo arenoso ter menor fertilidade provavelmente comprometeu o desenvolvimento normal da cultura, resultando em menor produtividade.

Por outro lado, o solo arenoso poderia facilitar uma maior disponibilidade do inseticida para absorção das plantas, em razão da menor adsorção junto ao solo (Rodríguez-Liébana et al., 2013; Tabassum et al., 2014). Embora ainda exista uma lacuna de informações sobre este tema, Sanchotene et al. (2010) diagnosticaram que em solos arenosos ocorre uma maior disponibilidade de herbicidas em TS de arroz irrigado, quando comparado com solos arenosos. Visando o controle de pragas iniciais, o emprego do inseticida clotianidina (neonicotinoide) em TS de milho para o controle do desfolhador Tanymecus dilaticollis (Coleoptera: Curculionidae) foi mais eficiente em condição de solos arenosos do que argilosos (Keszthelyi et al., 2008). Entretanto, o presente trabalho demonstrou que o solo arenoso foi o que apresentou o pior desenvolvimento das plantas, indicando que, além da mortalidade da praga e das injúrias foliares, outros parâmetros da cultura devem ser considerados, para evitar subestimar a capacidade de dano da praga.

De maneira geral, este trabalho comprova que para um adequado manejo de $D$. melacanthus é fundamental que seja considerado o tipo de solo em que a lavoura de milho será cultivada, pois este fator tem grande relevância sobre a eficiência no controle da praga. Desse modo, este estudo demonstra que para o estabelecimento do nível de dano de D. melacanthus em milho a condição edáfica local deve ser considera- da, em razão da variabilidade de respostas das plantas ao ataque da praga, de modo a permitir a adequação de diferentes estratégias de controle, de acordo com as condições locais de cultivo.

\section{Conclusões}

A eficiência do tratamento de sementes de milho com tiametoxam e imidacloprido + tiodicarbe é influenciada pelo tipo de solo, sendo que em solos de textura arenosa o desempenho desses inseticidas é prejudicado e estratégias de controle complementares podem ser necessárias.

\section{Agradecimentos}

Os autores agradecem às agências financiadoras Capes e CNPq, e ao auxílio estatístico da professora da Universidade Estadual de Londrina Dra. Inês Cristina Fonseca.

\section{Referências}

BRUSTOLIN, C.; BIANCO, R.; NEVES, P. M. O. J. Inseticidas em pré e pós-emergência do milho (Zea mays L.), associados ao tratamento de sementes, sobre Dichelops melacantus (Dallas) (Hemiptera: Pentatomidae). Revista Brasileira de Milho e Sorgo, Sete Lagoas, v. 10, n. 3, p. 215-223, 2011.

DOI: 10.18512/1980-6477/rbms.v10n3p215-223.

\section{CALIFORNIA DEPARTMENT OF PESTICEDE} REGULATION. Environmental fate of Imidacloprid. Sacramento, 2006.

CAMPBELL, S.; CHEN, L.; YU, J.; LI, Q. X. Adsorption and analysis of the insecticides thiamethoxam and indoxacarb in Hawaiian soils. Journal of Agricultural and Food Chemistry, Easton, v. 53, n. 13, p. 5373-5376, 2005. DOI: $10.1021 / \mathrm{jf0} 050272 \mathrm{r}$. 
CARBO, L.; MARTINS, E. L.; DORES, E. F.; SPADOTTO, C. A.; WEBER, O. L.; DE-LAMONICAFREIRE, E. M. Acetamiprid, carbendazin, diuron and thiamethoxam sorption in two Brazilian tropical soils. Journal of Environmental Science and Health Part B, New York, v. 42, n. 5, p. 499-507, 2007.

DOI: $10.1080 / 03601230701389439$.

CHOCOROSQUi, V. R. Bioecologia de espécies de Dichelops (Diceraeus) (Heteroptera: Pentatomidae) e danos em soja, milho e trigo no Norte do Paraná. 2001. 158 f. Tese (Doutorado em Entomologia) - Universidade Federal do Paraná, Curitiba, 2001.

CHOCOROSQUI, V. R.; PANIZZI, A. R. Impact of cultivation systems on Dichelops melacanthus (Dallas) (Heteroptera: Pentatomidae) populations and damage and its chemical control on wheat. Neotropical Entomology, Londrina, v. 33, n. 4, p. 487-492, 2004.

DOI: 10.1590/S1519-566X2004000400014.

CHOCOROSQUI, V. R.; PANIZZI, A. R. Nymph and adult biology of Dichelops melacanthus (Dallas) (Heteroptera: Pentatomidae) feeding on cultivated and non-cultivated host plants. Neotropical Entomology, Londrina, v. 37, n. 4, p. 353-360, 2008.

DOI: 10.1590/S1519-566X2008000400001.

KESZTHELYI, S.; KURUCSAI, P.; SZABÓ, T.; PÁLFÁM, F.; MARCZALI, Z. Chemical protections against corn weevil (Tanymecus dilaticollis Gyll.) on different soil types. Cereal Research Communications, Szeged, v. 36, p. 191-194, 2008. Supplement 5.

KODEŠOVÁ, R.; KOČÁREK, M.; KODEŠ, V.; DRÁBEK, O.; KOZÁK, J.; HEJTMÁNKOVÁ, K. Pesticide adsorption in relation to soil properties and soil type distribution in regional scale. Journal of Hazardous Materials, Amsterdam, v. 186, n. 1, p. 540-550, 2011.

DOI: $10.1016 /$ j.jhazmat.2010.11.040.

RITCHIE, S.; HANWAY, J. J. How a corn plant develops. Ames: Iowa State University of Science and Technology, 1989. (Special Report, n. 48).
RODRIGUES, R. B. Danos do percevejo-barrigaverde Dichelops melacanthus (Dallas, 1851) (Hemiptera: Pentatomidae) na cultura do milho. 2011. 105 f. Dissertação (Mestrado em Produção Vegetal) - Universidade Federal de Santa Maria, Santa Maria, 2011.

RODRÍGUEZ-LIÉBANA, J. A.; MINGORANCE, M. D.; PEÑA, A. Pesticide sorption on two contrasting mining soils by addition of organic wastes: effect of organic matter composition and soil solution properties. Colloids and Surfaces A: Physicochemical and Engineering Aspects, Amsterdam, v. 435, p. 71-77, 2013.

DOI: 10.1016/j.colsurfa.2012.12.027.

ROZA- GÓMES, M. F.; SALVADORI, J. R.; PEREIRA, P. R. V. da S.; PANIZZI, A. R. Injúrias de quatro espécies de percevejos pentatomídeos em plântulas de milho. Ciência Rural, Santa Maria, v. 41, n. 7, p. 1115-1119, 2011.

DOI: $10.1590 / \mathrm{S} 0103-84782011005000081$.

SANCHOTENE, D. M.; KRUSE, N. D.; AVILA, L. A.; MACHADO, S. L. O.; NICOLODI, G. A.; DORNELLES, S. H. B. Efeito do protetor dietholate na seletividade de clomazone em cultivares de arroz irrigado. Planta Daninha, Rio de Janeiro, v. 28, n. 2, p. 339-346, 2010. DOI: $10.1590 / \mathrm{S} 0100-83582010000200013$.

SANTOS, H. G. dos; JACOMINE, P. K. T.; ANJOS, L. H. C. dos; OliVEIRA, V. A. de; OLIVEIRA, J. B. de; COELHO, M. R.; LUMBRERAS, J. F.; CUNHA, T. J. F. (Ed.). Sistema brasileiro de classificação de solos. 2. ed. Rio de Janeiro: Embrapa Solos, 2006. 306 p.

TABASSUM, N.; RAFIQUE, U.; BALKHAIR, K. S.; ASHRAF, M. A. Chemodynamics of methyl parathion and ethyl parathion: adsorption models for sustainable agriculture. BioMed Research International, v. 2014, p. $1-8,2014$.

DOI: $10.1155 / 2014 / 831989$.

UNIVERSITY OF HERTFORDSHIRE. Agriculture and Environment Research Unit Pesticide properties database: april 2013 version. Hertfordshire, 2013. 